\title{
Temporal Changes in Genetic Diversity of Plasmodium Falci- parum Populations in Isolates from Asymptomatic Children in Brazzaville after the Introduction of ACT
}

\section{Research Article}

Volume 2 Issue 1- 2021

\begin{abstract}
Author Details
Dominique Fatima Voumbo-Matoumona ${ }^{1}$, Jean Akiana ${ }^{1}$, Chyvanelle Akiridzo-Ndous ${ }^{1}$, Lady Charlène Kouna ${ }^{2}$, Georcil Prosse Delore Ahouet Gnabang ${ }^{1}$, Jean Bernard Lekana-Douki ${ }^{3 *}$

${ }^{1}$ Departments of Masters/Licenses, University Marien Ngouabi, Congo

${ }^{2}$ Evolution, Epidemiology and Parasitic Resistances Unit [UNEEREP], Franceville Interdisciplinary Center for Medical Research, Gabon

${ }^{3}$ Parasitology, Mycology and Tropical Medicine Department, University of Health Sciences, Gabon
\end{abstract}

*Corresponding author

Jean Bernard Lekana-Douki, Lecturer, at the Parasitology, Mycology and Tropical Medicine Department, University of Health Sciences, PO Box: 4005 Libreville, Gabon, Evolution, Epidemiology and Parasitic Resistances Unit [UNEEREP], Interdisciplinary Center for Medical Research of Franceville, PO Box: 769, Franceville, Gabon

Article History

Received: December 13, 2020 Accepted: January 08, $2021 \quad$ Published: January 12, 2021

\begin{abstract}
Asymptomatic infections and genetic diversity of Plasmodium falciparum play a major role in the natural acquisition of immunity to malaria. This study investigated the genetic diversity of $m s p 1, m s p 2$ and glurp in P. falciparum isolate from patients in Republic of Congo. The use of ACT selected different populations of P. falciparum between 2010 and 2018. This change in the parasites needs to be monitored to prevent a re-increase of malaria prevalence despite the use of ACT.
\end{abstract}

Keywords: Asymptomatic malaria; Genetic diversity; Molecular markers; Pfmsp1; Pfmsp2; Pfglurp; Brazzaville

\begin{abstract}
Abbreviations: ACT: Artemisinin-Based Combination Therapy; P: Plasmodium; Pfmsp1: P. Falciparum Merozoite Surface Protein 1; Pfmsp2: P. Falciparum Merozoite Surface Protein 2; Pfglurp: P. Falciparum Glutamate-Rich Protein.
\end{abstract}

\section{Introduction}

Malaria is the most lethal parasitic infection and constitutes one of the major public health issues in the world, especially in subSaharan Africa where this disease is endemic and the transmission perennial [1]. The world malaria report registered 229 million cases and 409,000 deaths in 2019 [2]. Plasmodium falciparum is the most virulent malaria species and is responsible for more than $80 \%$ of all cases [2]. Asymptomatic malaria infection is defined by the presence [microscopic or submicroscopic] of malaria parasites in the human body without symptoms of uncomplicated or complicated malaria disease. Asymptomatic people are considered a great reservoir for malaria parasites and an important factor of multiplication and spread. These cases are often missed or underestimated because the control programs are focused on the passive and active study of symptomatic cases.

Genetic diversity of $P$. falciparum plays a major role in the natural acquisition of immunity to malaria infections and is also a concern to the development and deployment of control measures. In people living in malaria-endemic regions, immunity to $P$. falciparum is acquired as a result of natural exposure to multiple infections by different parasite strains over many years $[3,4]$. Asexual blood stages antigens, such as Merozoites Surface Protein-1 [MSP-1], merozoites surface protein-2 [MSP-2] and Glutamate-Rich Protein [GLURP] are considered prime candidates for the development of malaria vaccines and are also suitable markers for the identification of genetically distinct $P$. falciparum parasite subpopulations [3,5]. Block 2 of $P$. falciparum msp1 may be divided as the most polymorphic block with three [3] allelic families K1, Ro33 and Mad20. The P. falciparum msp 2 can be divided into two [2] allelic families FC27 and 3D7. The P. falciparum glurp is constituted by only one allelic family but remains highly polymorphic [6]. Several studies have reported that the frequencies of Pfmsp1, $P f m s p 2$ and Pfglurp allelic variants vary in different geographical areas, even in neighboring villages $[4,7]$. P. falciparum subpopulations vary also with clinical status or the presence of sickle cell trait [7-10].

The Republic of Congo is located in central Africa where malaria is endemic, and the transmission is perennial. During 2016, WHO estimated that almost a quarter of the Congolese population was at risk for malaria but only 171,847 cases were confirmed, and all confirmed cases were due to $P$. falciparum [11]. To control effectively malaria in the Republic of Congo, many malarial control measures have been deployed since the 2000s, including Artemisinin-Based Combination Therapy $[\mathrm{ACT}]$, Intermittent Preventive Treatment [IPT] and Long- 
Lasting Insecticide Nets [LLINs] for all pregnant women [12]. Before the change of policy, studies described the genetic diversity of $m s p 1$ and $m s p 2$ of $P$. falciparum populations from symptomatic patients collected in Brazzaville [13,14]. Less than ten[10] genotypes were found for each allelic family of $m s p 1$ [8, 6 and 1 for K1, Mad20 and Ro33 respectively] although ten genotypes were found for each allelic family of Pfmsp2 [3D7 and FC27] [13]. The mean of the Multiplicity of Infection [MOI] was 2-2.17 for Pfmsp1 and 1.7 for Pfmsp2 [13,14]. After the change of policy, only the genetic diversity of Pfmsp2 was analyzed also on isolates from symptomatic patients in the Republic of Congo $[10,15,16]$. One study compared the genetic diversity of $P f m s p 2$ in isolates between protected or asymptomatic individuals and unprotected or symptomatic patients with samples collected in 2010 [15]. The genetic diversity was higher in the protected group with 7 and 6 alleles although there were only 2 and 3 alleles for FC27 and 3D7 family respectively. However, the multiplicity of infection was higher in the unprotected group [1.43] than in the protected group [1.29] in 2015. Since the implementation of ACT, no studies assessed the polymorphism of Pfmsp1. Few data on P. falciparum diversity are available for isolates collected from asymptomatic patients.

This study aimed to investigate the genetic diversity of $m s p 1, m s p 2$ and glurp in Plasmodium falciparum isolates from asymptomatic patients from Brazzaville in the Republic of Congo four and thirteen years after the implementation of ACT.

\section{Methods and Materials}

\section{Ethics approval and participation consent}

This study was reviewed and approved by the Ethical Committee of the Health Research Ministry of the Republic of the Congo [no 007/DGRST/CERSSA and 008/MRSIT/IRSSA/CERSSA]. We used archived material from previous studies preserved for research purposes which were collected from the study populations composed of minors with the consent of legal tutor, respectively.

\section{Sample collection and diagnosis}

Samples used for this work were collected and used for previous studies. Transversal passages were done in health centers to collect peripheral blood from asymptomatic children under 15 years in 2010 and 2018 in Brazzaville in the south of the Republic of the Congo. In this study, only positive samples were considered. The diagnosis of $P$. falciparum infection was done by blood smears. A total of 33 and 37 isolates infected by Plasmodium falciparum were collected.

\section{DNA extraction}

DNA was extracted with the DNA Blood Omega Bio-Tek E.Z.N.A ${ }^{\circ}$ method [Omega Bio-Tek, USA] as previously described [17]. In short, $250 \mu \mathrm{l}$ of blood, $25 \mu \mathrm{l}$ of protease $\mathrm{K}[20 \mathrm{mg} / \mathrm{ml}]$ and $250 \mu \mathrm{l}$ of lysis buffer were mixed and heated to $71^{\circ} \mathrm{C}$ for 45 minutes, and then $260 \mu \mathrm{l}$ of isopropanol was added. This mixture was transferred to a column and centrifuged at $8000 \mathrm{~g}$ for 1 minute. The column was washed twice, and DNA was eluted with $100 \mu \mathrm{l}$ of sterile water preheated to $70^{\circ} \mathrm{C}$. DNA aliquots were kept at $-20^{\circ} \mathrm{C}$ until use.

\section{Genetic diversity}

The $P$. falciparum genes of merozoites surface protein 1 and 2 [ $m s p 1$ and $m s p 2$ ] and the glutamate-rich protein [glurp] sequences were amplified. Electrophoresis on agarose gel was done to determine the size of each fragment. The reaction mixture and conditions for the thermal cycler and electrophoresis were done according to the WWARN protocols. Primers used are described in table 1. The polymorphism in each allelic family was analyzed; assuming that one band represented one amplified PCR fragment derived from a single copy of $P$. falciparum msp 1, msp 2 or glurp genes. Alleles in each family were considered the same if fragment size were within $20 \mathrm{bp}$ interval. The minimum number of genotypes per isolate was estimated to be the highest number of fragments identified for either $m s p 1, m s p 2$ or glurp.

\section{Statistical analysis}

Data were recorded in Excel [Office 2010]. Statistical analysis was carried out with the software R version 3.2.1. Ages were expressed as medians and Interquartile Ranges [IQR]. The $m s p 1, m s p 2$ or glurp allele frequency was calculated as the proportion of allele found for the allelic family out of the alleles detected in isolates. The Multiplicity of Infection [MOI] was defined as the minimum number of $P$. falciparum genotypes per infected subject and estimated by dividing the number of amplified PCR fragments reflecting parasite genotypes by the number of positive samples. The Chi-square test was used to compare categorical variables among groups. The nonparametric Kruskal Wallis, Student t-test and Fisher's exact test were used for group comparisons, as appropriate. Significance was assumed at $p<0.05$.

Table 1: Primers and size range of amplicons.

\begin{tabular}{|c|c|c|}
\hline Genes & PCR/Allelic Family & Primers \\
\hline \multirow{3}{*}{ Pfmspl } & PCR1 & $\begin{array}{l}\text { 5' CTA GAA GCT TTA GAA GAT GCA GTA TTG 3' } \\
\text { 5' AAA TAG TAT TCT AAT TCA AGT GGA TCA 3' }\end{array}$ \\
\hline & PCR2/Mad20 & $\begin{array}{l}\text { 5' AAA TGA AGG AAC AAG TGG AAC AGC TGT TAC 3' } \\
5^{\prime} \text { ATC TGA AGG ATT TGT ACG TCT TGA ATT ACC 3' }\end{array}$ \\
\hline & PCR2/Ro33 & $\begin{array}{l}\text { 5' TAA AGG ATG GAG CAA ATA CTC AAG TTG TTG 3' } \\
\text { 5' CAT CTG AAG GAT TTG CAG CAC CTG GAG ATC 3' }\end{array}$ \\
\hline \multirow{3}{*}{ Pfmsp2 } & PCR1 & $\begin{array}{l}\text { 5' AAA TGA AGG AAC AAG TGG AAC AGC TGT TAC 3' } \\
5^{\prime} \text { ATC TGA AGG ATT TGT ACG TCT TGA ATT ACC 3' }\end{array}$ \\
\hline & PCR2/3D7 & $\begin{array}{l}\text { 5' AAT ACT AAG AGT GTA GGT GCA A/GAT GCT CCA3' } \\
\text { 5' TTT TAT TTG GTG CAT TGC CAG AAC TTG AAC 3' }\end{array}$ \\
\hline & PCR2/FC27 & $\begin{array}{c}\text { 5' ATG AAG GTA ATT AAA ACA TTG TCT ATT ATA 3' } \\
\text { 5' CTT TGT TAC CAT CGG TAC ATT CTT 3' }\end{array}$ \\
\hline \multirow[t]{2}{*}{ Pfglurp } & PCR1 & $\begin{array}{l}\text { 5' TGA AAT TGA AGA TGT TCA CAC TGA AC 3' } \\
\text { 5' GTG GAA TTG CTT TTT CTT CAA CAC TAA 3' }\end{array}$ \\
\hline & PCR2 & $\begin{array}{c}\text { 5' TGT TCA CAC TGA ACA ATT AGA TTT AGA TCA 3' } \\
\text { 5' GTG GAA TTG CTT TTT CTT CAA CAC TA 3' }\end{array}$ \\
\hline
\end{tabular}




\section{Results}

\section{Plasmodium falciparum merozoites surface protein 1} [Pfmsp1]

Approximately $90.90 \%$ [ $\mathrm{n}=30]$ and $64.86 \%$ [ $\mathrm{n}=24]$ of isolates could be amplified for Pfmsp1 for 2010 and 2018 respectively.

The overall MOI associated with the polymorphism of Pfmsp1 decreased slowly from 2.7 to 2.04 between 2010 and 2018 [ $p=0.14$ ] The MOI associated with the Mad 20 family was 1.83 and 1.14 for 2010 and 2018 respectively [ $p=0.12$ ]. The MOI associated with the Ro33 family was 2.6 and 1.89 for 2010 and 2018 respectively [ $p=0.33$ ]. The frequencies of isolates with multiple allelic types were not significantly different during the time [Table 2] [p>0.05]. In 2010, 7 isolates carried the Mad20 and Ro33 types although 4 isolates were found with both types in 2018 [ $p=0.74$ ].

We investigated the polymorphism of the allelic families Ro33 and Mad20 of Pfmsp1. The proportion of the family Ro33 was predominant in the two years: $74.71 \%$ in 2010 vs $80.95 \%$ in 2018 and the proportion of Mad20 was 25.29 and 19.05 for the same years. No difference was observed in the proportion of Mad20 and Ro33 [ $p=0.57]$.

Between 2010 and 2018, the number of alleles decreased from 28 to 16 [about $43 \%$ decline] for Ro33 and from 15 to 5 [about $67 \%$ decline] for Mad20. The band sizes were 100-480 bp for 2010 and 100-220 for 2018 for the Mad20 type; 100-950 bp for 2010 and 100-420 for 2018 for the Ro33 type [Figure 1]. Only 10 alleles of Ro33 and 3 alleles of Mad 20 were common in the two years.

\section{Plasmodium falciparum merozoite surface protein 2 [Pfmsp2]}

Approximately $63.64 \%$ [ $\mathrm{n}=21]$ and $78.38 \%$ [ $\mathrm{n}=29]$ of isolates could be amplified for Pfmsp2 for 2010 and 2018 respectively.

The overall MOI associated with the polymorphism of Pfmsp2 was similar [1.95 to 1.93 ] between 2010 and 2018 [ $p=0.94]$. The MOI associated with the 3D7 family increased from 1 to 1.22 between 2010 and 2018 respectively [ $p=0.04]$. The MOI associated with the Fc27 family was 1.72 and 1.43 for 2010 and 2018 respectively [ $p=0.34$ ]. The frequencies of isolates with multiple allelic types were not significantly different during that time [table 2] [ $p>0.05]$. In 2010, 7 isolates carried the Fc27 and 3D7 types whereas 12 isolates were found with both types in 2018 [ $p=0.85]$.

We investigated the polymorphism of the allelic families 3D7 and Fc27 of Pfmsp2. The proportion of the family Fc27 was predominant in the two years: $74.36 \%$ in 2010 vs $65.38 \%$ in 2018 and the proportion of 3D7 was 25.64 and 34.62 for the same years. No difference was observed in the proportion of 3D7 and Fc27 [ $p=0.49]$.

Between 2010 and 2018, the number of alleles increased from 8 to 15 [about $87.5 \%$ rise] for Fc27 and from 6 to 9 [about 50\% rise] for 3D7. The band sizes were $390-560 \mathrm{bp}$ for 2010 and 250-460 for 2018 for the 3D7 type; 480-680 bp for 2010 and 100-710 for 2018 for the Fc27 type [Figure 2]. Only one allele of 3D7 was common in the two years. No common alleles were observed.

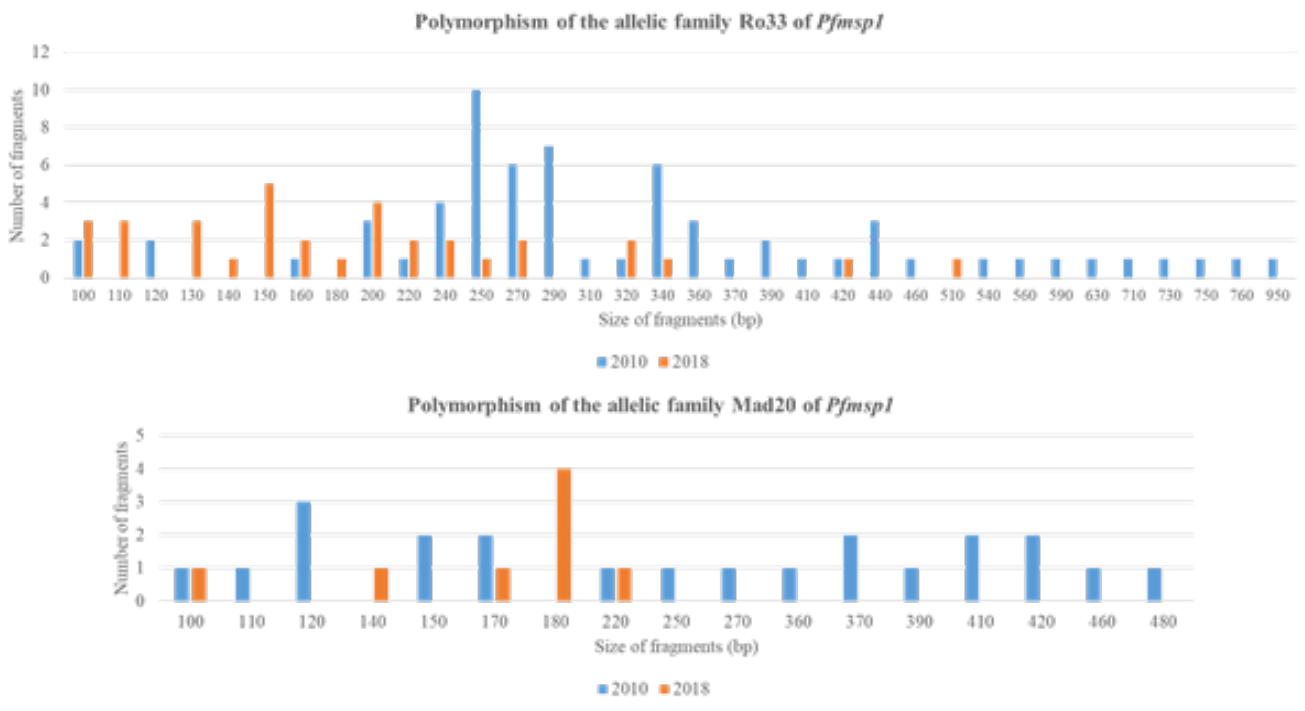

Figure 1: Polymorphism of Pfmsp1.

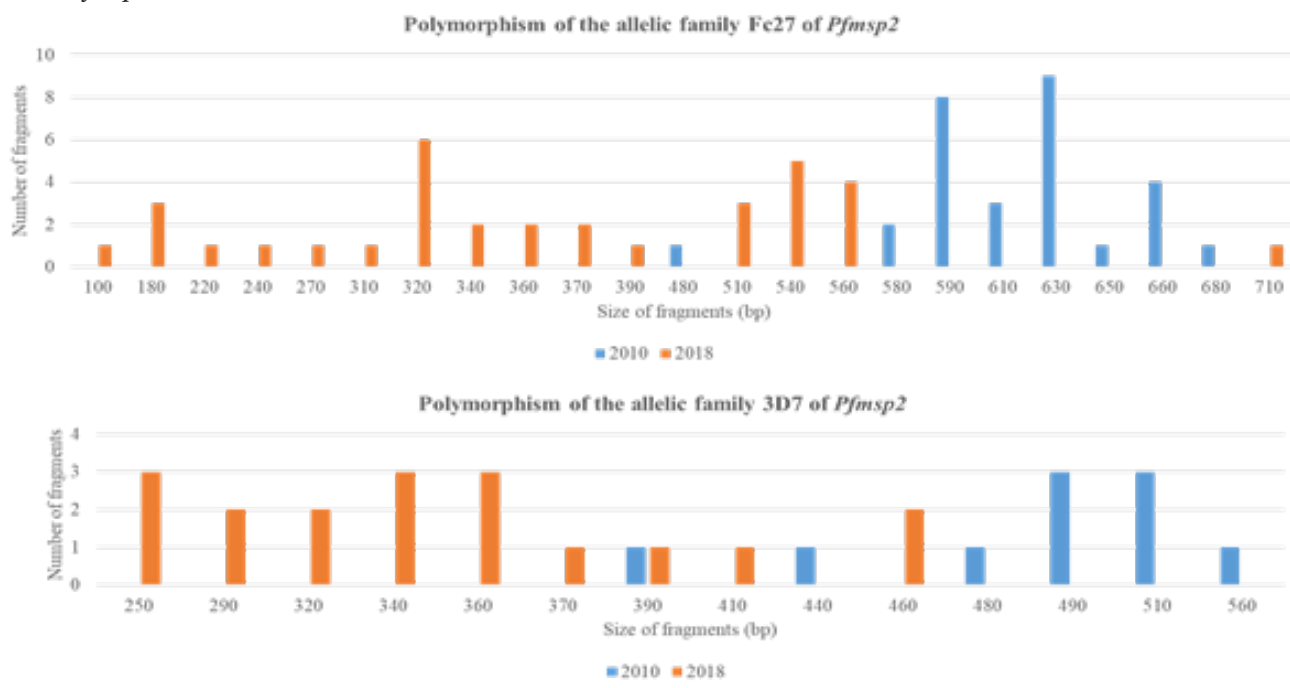

Figure 2: Polymorphism of Pfmsp2. 


\section{Plasmodium falciparum glutamate rich protein [Pfglurp]}

Approximately $81.82 \%$ [ $\mathrm{n}=27]$ and $70.27 \%$ [n=26] of isolates could be amplified for Pfglurp for 2010 and 2018 respectively.

The MOI associated with the polymorphism of Pfglurp was similar [1.76 to 1.57] between 2010 and 2018 [ $p=0.53$ ]. In 2010, isolates with only one allele of Pfglurp was major; in 2018, the number of isolates with two alleles was 5 -fold higher than previously observed [Table 2] [p>0.01].

Between 2010 and 2018, the number of alleles increased from 19 to 22 [about $15.79 \%$ rise]. The band sizes were $150-850$ bp for 2010 and $170-870$ for 2018 [Figure 3]. Only 9 alleles were common in the two years [Figure 3].

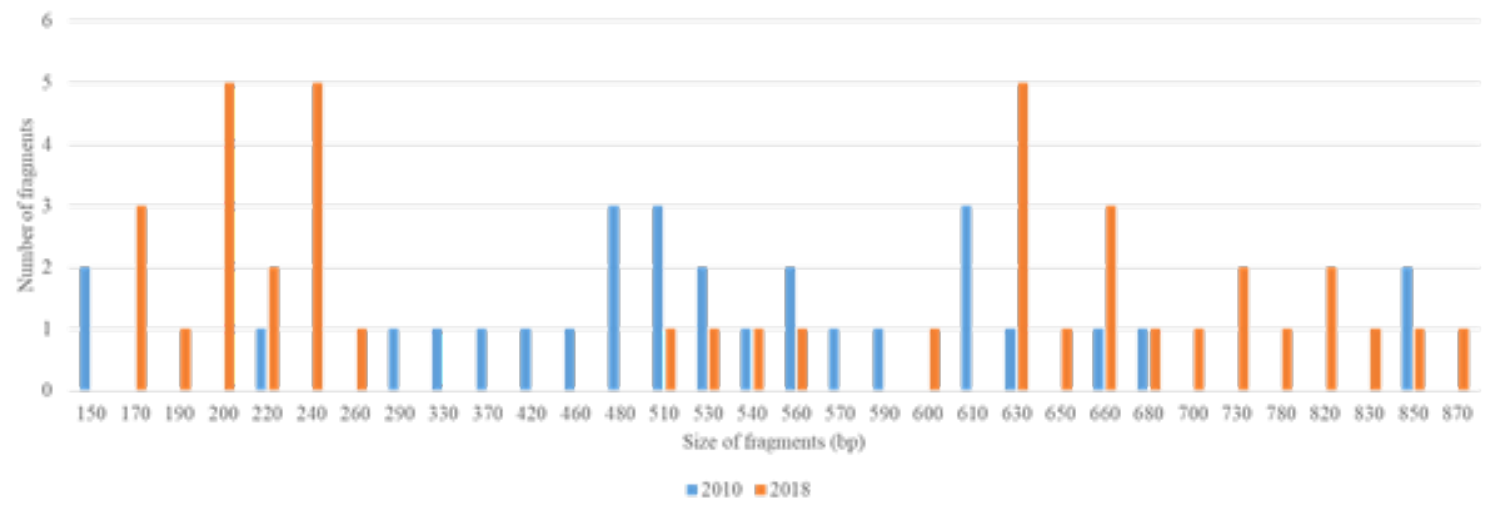

Figure 3: Polymorphism of Pfglurp.

Table 2: Minimal number of allele per sample.

\begin{tabular}{|c|c|c|c|c|c|}
\hline & Allelic Family & Number of Alleles Per Sample & $2010 \mathrm{n} / \mathrm{N}$ & $2018 \mathrm{n} / \mathrm{N}$ & $p$ \\
\hline \multirow{9}{*}{ Pfmspl } & \multirow{4}{*}{ Mad20 } & 1 & $6 / 11$ & $6 / 7$ & \multirow{4}{*}{0.85} \\
\hline & & 2 & $3 / 11$ & $1 / 7$ & \\
\hline & & 4 & $1 / 11$ & $0 / 7$ & \\
\hline & & 5 & $1 / 11$ & $0 / 7$ & \\
\hline & \multirow{5}{*}{ Ro33 } & 1 & $7 / 25$ & $11 / 18$ & \multirow{5}{*}{0.1} \\
\hline & & 2 & $8 / 25$ & $2 / 18$ & \\
\hline & & 3 & $7 / 25$ & $2 / 18$ & \\
\hline & & 4 & $0 / 25$ & $1 / 18$ & \\
\hline & & 5 & $3 / 25$ & $2 / 18$ & \\
\hline \multirow{6}{*}{$P f m s p 2$} & \multirow{4}{*}{ FC27 } & 1 & $9 / 18$ & $14 / 23$ & \multirow{4}{*}{0.8} \\
\hline & & 2 & $6 / 18$ & $7 / 23$ & \\
\hline & & 3 & $2 / 18$ & $2 / 23$ & \\
\hline & & 4 & $1 / 18$ & $0 / 23$ & \\
\hline & \multirow{2}{*}{$3 \mathrm{D} 7$} & 1 & $10 / 10$ & $14 / 18$ & \multirow{2}{*}{0.27} \\
\hline & & 2 & $0 / 10$ & $4 / 18$ & \\
\hline \multirow{4}{*}{ Pfglurp } & & 1 & $10 / 17$ & $11 / 26$ & \multirow{4}{*}{$5.37^{\star} 10^{\mathrm{e}}-3$} \\
\hline & & 2 & $3 / 17$ & $15 / 26$ & \\
\hline & & 3 & $3 / 17$ & $0 / 26$ & \\
\hline & & 4 & $1 / 17$ & $0 / 26$ & \\
\hline
\end{tabular}

Table 3: Proportion of allelic family of each gene per year.

\begin{tabular}{|c|c|c|c|c|}
\hline & \multicolumn{2}{|c|}{ Pfmsp1 N (\%) } & \multicolumn{2}{c|}{ Pfmsp2 N (\%) } \\
\hline & MAD20 & RO33 & FC27 & 3D7 \\
\hline 2010 & $22(25,29)$ & $65(74,71)$ & $29(74,36)$ & $10(25,64)$ \\
\hline 2018 & $8(19,05)$ & $34(80,95)$ & $34(65,38)$ & $18(34,62)$ \\
\hline$p$ & \multicolumn{2}{|c|}{0,57} & \multicolumn{2}{c|}{0,49} \\
\hline
\end{tabular}

\section{Discussion}

Among the most studied markers of Plasmodium falciparum diversity are Pfmsp1, Pfmsp2 and Pfglurp. These genes are highly polymorphic and thus constitute great markers to distinguish $P$. falciparum populations during infections. This work aimed to determine the evolution of the genetic diversity of these markers in isolates collected from asymptomatic children aged 0 to 15 years in Brazzaville in 2010 and 2018. This study is among the few that address allelic diversity in asymptomatic carrier individuals in Congo.
In this study, not all isolates gens could be genotyped. The use of lowquality stored DNA or primers, not sensitive enough could explain these success percentages.

The molecular analysis allowed us to explore the polymorphism of the Mad20 and Ro33 allelic families of Pfmsp1; FC27 and 3D7 from Pfmsp2 and Pfglurp, which are associated with the genetic diversity of Plasmodium falciparum. Overall, we have demonstrated a decrease in the allelic diversity studied between 2010 and 2018 for $P f m s p 1$ and an increase in allelic diversity for $P f m s p 2$ and Pfglurp. A decrease in band 
size of alleles found for Pfmsp1 and Pfmsp2 and an increase in band size of Pfglurp alleles were also observed. This could be the result of the pressure from the drug with the intensification of the fight against malaria, particularly that of ACTs as observed in Comoros [18]. In the Republic of Congo, less attention has been paid to studying the genetic diversity of $P$. falciparum among of asymptomatic parasites carriers. In 2015, almost 10 years after the introduction of ACTs, the multiplicity of infection found in our study was similar to that described previously for Pfmsp1 [2.5] but greater than that described for Pfmsp2 [1.59] in Congo for children who consulted the pediatrics department in Brazzaville after microscopic diagnosis $[10,19]$. These MOIs are greater than those described for these markers before the introduction of ACTs in the south of Brazzaville [13,14]. These genes appeared to be highly influenced by the treatment and other malaria measures in survival processes [20,21]. For Pfmsp1 the number of subpopulations per infection did not change whereas the genetic diversity decrease. To adapt itself efficiently, changes are more expressed in Pfmsp2 and Pfglurp, for which the parasite is increasing the number of subpopulations per infection and the genetic diversity of allelic family. In this work, new alleles of these markers were found with new bands size.

The isolates analyzed for this study carried multiple parasite populations, up to at least five allelic populations per gene considered. Although the polymorphism of the K1 allelic family has not been studied, the proportion of Ro33 is greater than that of Mad20 in isolates from individuals and asymptomatic for Pfmsp 1; a similar observation is made for the FC27 family of Pfmsp2 as previously described for isolates from symptomatic patients in Brazzaville [10,19]. However, the number of Ro33 and Mad20 alleles for Pfmsp1 and Fc27 and 3D7 for Pfmsp2 found here in asymptomatic patients is higher than that observed in symptomatic patients before and after the introduction of ACTs $[8,10,13,16,19]$. This observation can be extended to the numbers of fragments of different sizes. This difference could be explained by the fact that not all Plasmodium strains are equal in terms of induction of the pathology. One can easily imagine that many strains found in asymptomatic patients are not virulent, whereas, in symptomatic patients, a few strains emerge and induce febrile access. The proportions found in our study can also be compared to what has been described in patients consulting in northern Ethiopia and Eritrea [22]. The Republic of Congo is considered a highly endemic country for malaria with a high level of malaria transmission. Studies conducted in low transmission areas show that the genetic diversity of $P f m s p 1$ and $P f m s p 2$ is low contrary to what used to be observed in high transmission areas, where genetic diversity is also high $[20,21,23,24]$.

This study is the first to explore the diversity of the Pfglurp marker in Congo. However, in comparison to isolates from patients consulting in northern Ethiopia, we observe that the number of Pfglurp alleles found here is higher than that described in malaria symptomatic patients [22]. This confirms the genetic diversity of parasitic populations depending on the geographical location, the level of transmission of the parasite and the presence of the clinical symptoms of the disease $[7,25,26]$. In this study, despite the significant decrease in populations per infected isolates, the number of alleles increased.

The absence of Pfmsp1-K1 allelic family is a limitation to explore the genetic diversity of this gene. Agarose gel electrophoresis of amplified products lacks sufficient sensitivity to resolve a very small size difference between different bands, which constitutes a limitation of the study. Alternative methods such as genotyping using capillary electrophoresis or genome sequencing are preferred and would offer a better resolution to distinguish parasite populations.

\section{Conclusion}

These results showed that the diversity of the Pfmspl has diminished whereas the diversity of Pfmsp2 and Pfglurp genes has increased over the years. This could be seen as a sign that $P$. falciparum is adapting to the pressure of established malaria control methods.

\section{Acknowledgment}

We thank the patients and the staff of the medical centers from whom the isolates were collected in Brazzaville in 2010 and 2018. We also thank all the people who contributed to the logistics of this study.

\section{Funding}

This study was supported by funds from the Laboratoire National de Santé Public du Congo, the Gabonese government, Total Gabon, the Centre International de Recherches Médicales de Franceville, the Agence Universitaire de la Francophonie and the Associations of African Universities.

\section{Conflict of Interest}

Author has no conflict of interest.

\section{References}

1. WHO (2016) World malaria report

2. WHO (2020) World malaria report

3. Kiwanuka GN (2009) Genetic diversity in Plasmodium falciparum merozoite surface protein 1 and 2 coding genes and its implications in malaria epidemiology: A review of published studies from 1997-2007. J Vector Borne Dis. 46(1): 1-12.

4. Zwetyenga J, Rogier C, Tall A, Fontenille D, Snounou G (1998) No Influence of Age on Infection Complexity and Allelic Distribution in Plasmodium Falciparum Infections in Ndiop, a Senegalese Village With Seasonal, Mesoendemic Malaria. Am J Trop Med Hyg 59(5): 726-735.

5. Theisen M, Adu B, Mordmüller B, Singh S (2017) The GMZ2 malaria vaccine: from concept to efficacy in humans. Expert Rev Vaccines 16(9): 907-917

6. B H, R T, IS Z, C B, Borre M (1993) Glutamate rich Plasmodium falciparum antigen (GLURP). Parasitologica 47-50.

7. Wanji S, Kengne-ouafo AJ, Eyong EEJ, Kimbi HK, Tendongfor N, et al. (2012) Genetic Diversity of Plasmodium falciparum Merozoite Surface Protein-1 Block 2 in Sites of Contrasting Altitudes and Malaria Endemicities in the Mount Cameroon Region. Am J Trop Med Hyg 86(5): 764-774

8. Ibara-okabande R, Koukouikila-koussounda F, Ndounga M, Vouvoungui J, Malonga V, et al. (2012) Reduction of multiplicity of infections but no change in msp2 genetic diversity in P. falciparum isolates from Congolese children after introduction of artemisinincombination therapies. Malar J 11(Suppl 1): P70.

9. Abiodun IB, Oluwadun A, Ayoola AO (2014) Plasmodium falciparum Merozoite Surface Protein-1 Polymorphisms among Asymptomatic Sickle Cell Anemia Patients in Nigeria. Acta medica Iranica 54(1): 4453.

10. Etoka-beka MK, Ntoumi F, Kombo M, Deibert J, Poulain P (2016) Plasmodium falciparum infection in febrile Congolese children: prevalence of clinical malaria 10 years after introduction of artemisinincombination therapies. Trop Med Int Health 21(12): 1496-1503.

11. WHO (2017) World Malaria Report.

12. Ministère de la Santé et de la Population, Congo R du. PSN 2014-2018_ CGO_final.

13. Mayengue PI, Ndounga M, Malonga FV, Bitemo M, Ntoumi F (2011) Genetic polymorphism of merozoite surface protein-1 and merozoite surface protein-2 in Plasmodium falciparum isolates from Brazzaville, Republic of Congo. Malar J 10(1): 276.

14. Tsumori Y, Ndounga M, Sunahara T, Hayashida N, Inoue M, et al. (2011) Plasmodium falciparum: Differential selection of drug resistance alleles in contiguous urban and peri-urban areas of Brazzaville, Republic of Congo. PLoS One 6(8). 
15. Ibara-Okabande R, Koukouikila-Koussounda F, Ndounga M, Vouvoungui J, Malonga V, et al. (2012) Reduction of multiplicity of infections but no change in msp2 genetic diversity in Plasmodium falciparum isolates from Congolese children after introduction of artemisinin-combination therapy. Malar J 11(1): 1.

16. Koukouikila-Koussounda F, Malonga V, Mayengue P, Ndounga M, Vouvoungui C, et al. (2012) Genetic polymorphism of merozoite surface protein 2 and prevalence of K76T pfcrt mutation in Plasmodium falciparum field isolates from Congolese children with asymptomatic infections. Malar J 11(1): 105.

17. Lekana-Douki JB, Boutamba SDD, Zatra R, Edou SEZ, Ekomy H, et al. (2011) Increased prevalence of the Plasmodium falciparum Pfmdr1 86N genotype among field isolates from Franceville, Gabon after replacement of chloroquine by artemether-lumefantrine and artesunate-mefloquine. Infect Genet Evol 11(2): 512-517.

18. Huang B, Tuo F, Liang Y, Wu W, Wu G, et al. (2018) Temporal changes in genetic diversity falciparum isolates from Grande Comore Island after introduction of ACT. Malar J 1-14.

19. Gueye NSG, Ntoumi F, Vouvoungui C, Kobawila SC, NKombo M, et al. (2018) Plasmodium falciparum merozoite protein-1 genetic diversity and multiplicity of infection in isolates from Congolese children consulting in a pediatric hospital in Brazzaville. Acta Trop 183: 78-83.

20. Gosi P, Lanteri C a, Tyner SD, Se Y, Lon C, et al. (2013) Evaluation of parasite subpopulations and genetic diversity of the msp1, msp2 and glurp genes during and following artesunate monotherapy treatment of Plasmodium falciparum malaria in Western Cambodia. Malar J 12(1): 403.

21. Razak MRMA, Sastu UR, Norahmad NA, Abdul-Karim A, Muhammad A, et al. (2016) Genetic Diversity of Plasmodium falciparum Populations in Malaria Declining Areas of. PLoS One 1-22.

22. Mohammed H, Kassa M, Mekete K, Assefa A, Taye G, et al. (2018) Genetic diversity of the msp-1, msp-2, and glurp genes of Plasmodium falciparum isolates in Northwest Ethiopia. Malar J 17(1): 386.

23. Sane R, Talla C, Diouf B, Sarr FD, Diagne N, et al. (2019) Low genetic diversity and complexity of submicroscopic Plasmodium falciparum infections among febrile patients in low transmission areas in Senegal. PLoS One 27: 1-14.

24. Soe TN, Wu Y, Tun MW, Xu X, Hu Y, et al. (2017) Genetic diversity of Plasmodium falciparum populations in southeast and western Myanmar. Parasites \& Vector 1-6.

25. Olasehinde GI, Yah CS, Singh R, Ojuronbge OO, Ajayi AA, et al. (2012) Genetic diversity of Plasmodium falciparum field isolates from southwestern Nigeria. Afr Health Sci 12(3): 355-361.

26. Mohammed H, Kassa M, Mekete K, Assefa A, Taye G, et al. (2018) Genetic diversity of the msp-1, msp-2, and glurp genes of Plasmodium falciparum isolates in Northwest Ethiopia 11 Medical and Health Sciences 1108 Medical Microbiology. Malar 17(1): 1-8. 